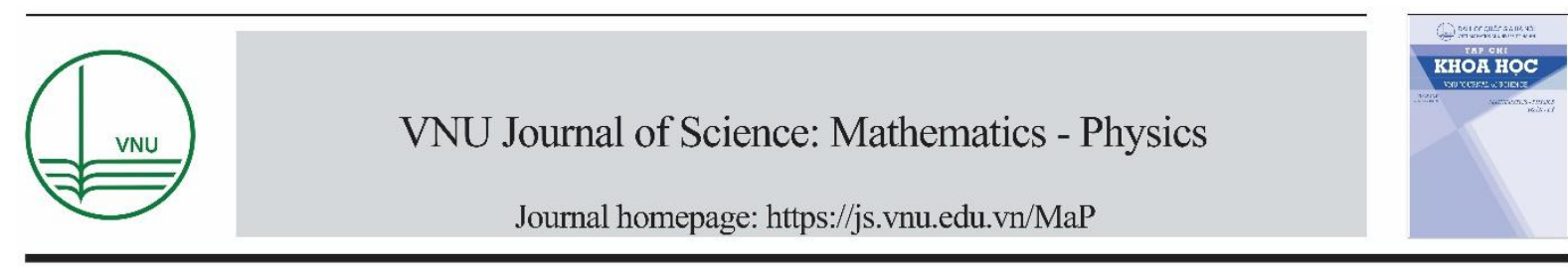

Original Article

\title{
Ovalbumin Based Microlasers
}

\author{
Hanh Hong Mai* \\ Faculty of Physics, University of Science, Vietnam National University, \\ 334 Nguyen Trai, Thanh Xuan, Hanoi, Vietnam
}

Received 02 April 2020

Revised 29 April 2020; Accepted 02 May 2020

\begin{abstract}
In this work, biomaterial based microlasers were successfully fabricated by a cost effective, simple and environmental friendly method. The as-fabricated microlasers were made of biocompatible material including ovalbumin doped Rhodamine B at low weight percentage. The lasers are shaped as solid-state spheres with various diameters ranging from $20 \mu \mathrm{m}$ to over $100 \mu \mathrm{m}$. It was shown that inside the spherical cavity which supported whispering gallery mode, a lasing emission was generated with a low threshold of $17.5 \mu \mathrm{J} / \mathrm{mm}^{2}$, and a high Q-factor of approximately 3000. The remarkable lasing properties of the dye doped ovalbumin microlasers are considered as important bases for their future biological and medical applications.
\end{abstract}

Keywords: Microlasers, ovalbumin, whispering gallery mode.

\section{Introduction}

Recently, microlasers have drawn tremendous attention due to their wide range applications in biology and medicine [1,2]. For biological and medical applications, biomaterials are preferably used fore both gain and laser cavity due to their biodegradable, bioresorbable, biocompatible, and typically environmentally friendly properties [3-5]. Ovalbumin (OVA) is the major protein from egg white, containing $54 \%(\mathrm{~m} / \mathrm{m})$ of the total protein content [6]. OVA has been used in numerous optical, electrical and medical applications. For example, OVA is used in immunological studies as a carrier protein in vaccines and a model protein in egg sensitivity allergy tests [7]. In optical applications, OVA is also a promising material due to its high solubility in aqueous media, good optical transparency. Despite these excellent properties, ovalbumin has been rarely investigated as an appropriate material for a laser cavity.

\footnotetext{
* Corresponding author.

Email address: hanhhongmai@hus.edu.vn
}

https//doi.org/ 10.25073/2588-1124/vnumap.4504 
To date, there has been variety of cavity structures which were successfully implemented in microlasers such as whispering gallery mode, distributed feedback, Fabry-Perot, and random cavity structures [8-10], [11], [12]. Among numerous cavity structures, the whispering gallery mode (WGM) cavity structure has shown outstanding performances in bio-sensing $[13,14]$ and cell tracking $[15,16]$ due to its remarkable properties of low lasing threshold, high Q-factor, small volume and simple fabrication.

In this work, ovalbumin microlaser based whispering gallery mode cavity structure were fabricated in a novel way using a simple and effective technique. The obtained ovalbumin microspheres can serve as high $\mathrm{Q}$ factor resonators. By doping organic dye molecules to these structures, a lasing emission was obtained under optical pumping. The lasing mechanism and lasing characteristics are also studied in this work.

\section{Experiment}

\subsection{Reagents and Chemicals}

Ovalbumin, Rohdamin B (RhB), and ethyl acetate $\left(\mathrm{CH}_{3} \mathrm{COOC}_{2} \mathrm{H}_{5}\right)$ were purchased from SigmaAldrich. Polydimethylsiloxane (PDMS) was purchased from Sylgard 184 Silicon Elastomer.

The $5 \mathrm{wt} \%$ OVA solution was prepared by dissolving in deionized water at room temperature. Afterward a proper volume of OVA solution was mixed with $1 \mathrm{wt} \% \mathrm{RhB}$ solution to obtain dye-doped OVA solution. This solution was then used for microsphere laser fabrication. The ratio of OVA and $\mathrm{RhB}$ in a microsphere laser is of $99.5 \mathrm{wt} \%$ and $0.5 \mathrm{wt} \%$.

\subsection{Fabrication Process}

The fabrication process of micro-biolaser based on dye-doped OVA solution is shown in Figure 1. A droplet of dye-doped OVA solution was drop cast into PDMS resin. A micro needle was used to split the former droplet in to smaller micro-size droplets. Since the dye-doped solution are undissolved in PDMS, the microspheres were self-assembly formed as a result of surface tension. The microspheres were then shrinked and solidified by gradually heating the PDMS up to $90^{\circ} \mathrm{C}$. Finally, the solidmicrospheres were collected by dissolving PDMS in ethyl acetate solution $\left(\mathrm{CH}_{3} \mathrm{COOC}_{2} \mathrm{H}_{5}\right)$, and kept in this solution for further optical measurements.

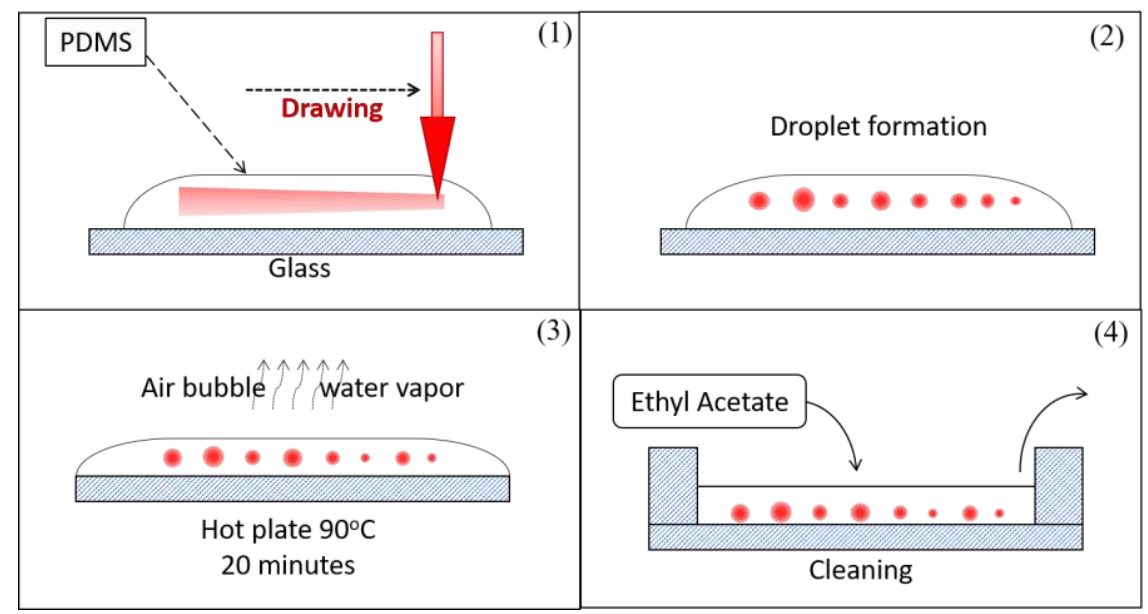

Figure 1. Schematic diagram of dye doped OVA microspheres fabrication process. 


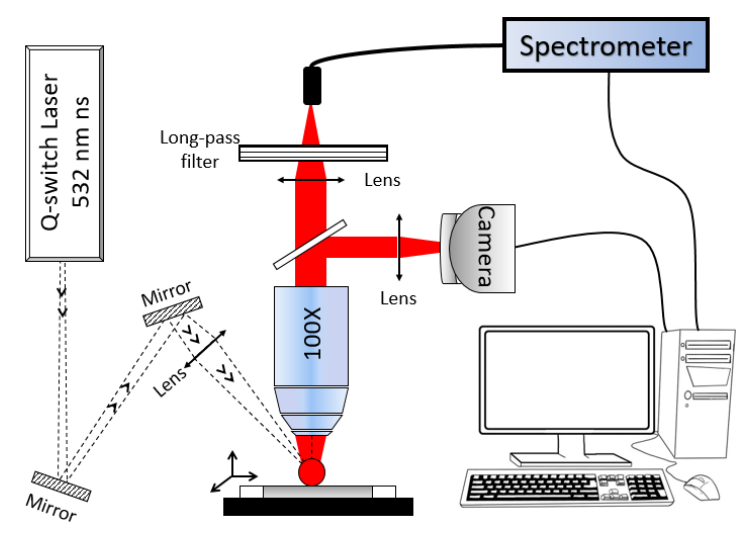

Figure 2. Schematic of the optical characterization setup.

\subsection{Optical characterization}

The lasing emission from each microsphere was investigated by a micro-photoluminescent setup at room temperature (Figure 2). The setup includes a $532 \mathrm{~nm}$ nanosecond Q-switch Nd:YAG laser (pulse width of 8ns) operated as an excitation source, a microscope for light collimation, a spectrometer (AvaSpec-2048L from Avantes), and a camera. The OVA microspheres, placed on a highly transparent slide of glass, were excited under different energy with repetition rate of $10 \mathrm{~Hz}$. Lasing emission was the collected by the objective lens ( $10 \mathrm{X}$ of magnification, $\mathrm{NA}=0.25)$ of the microscope, guided to the camera for top view image capture, and to the spectrometer for spectral analysis. The spectrometer's resolution is of approx. $0.2 \mathrm{~nm}$.

The surface morphologies of the as-prepared microspheres were investigated by a Scanning electron microscope (NOVA NANOSEM 450).

\section{Results and Discussion}

The optical image of the dye doped OVA microspheres demonstrates spherical shapes of the microlasers. The microspheres' diameter range from $30 \mu \mathrm{m}$ to $100 \mu \mathrm{m}$ (Figure 3a). The SEM image of a microsphere shows a smooth, round surface which ensures the WGM lasing emission quality (Figure 3b).
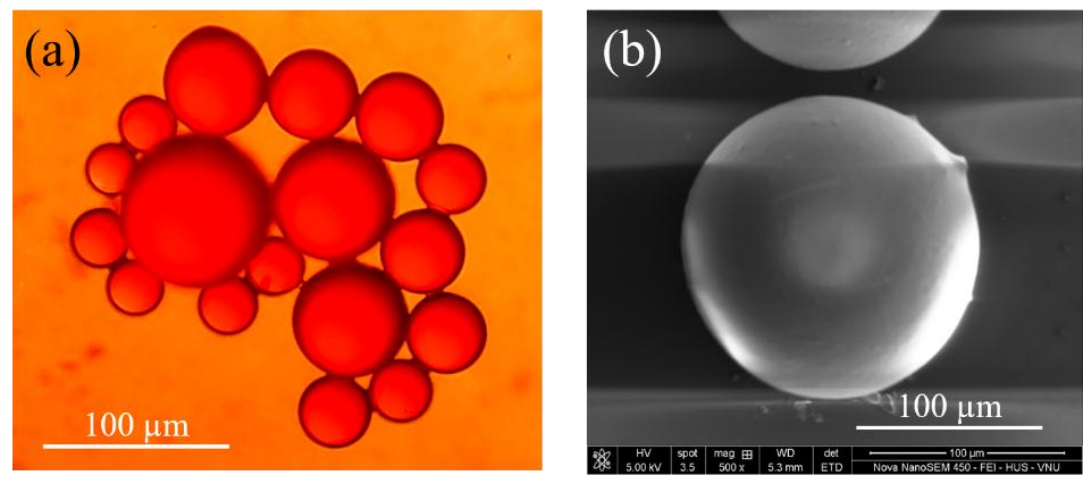

Figure 3. (a) Optical image of the dye-doped OVA microspheres

(b) SEM image of a dye doped OVA microsphere. The scale bars are $100 \mu \mathrm{m}$. 
Lasing emission was observed from individual dye-doped OVA microsphere under laser pulse pumping. Figure $4 \mathrm{a}$ presents the lasing performance of a microsphere which has a diameter of $\sim 44 \mu \mathrm{m}$. When the pump pulse energy exceeded $22.3 \mu \mathrm{J} / \mathrm{mm}^{2}$, multiple sharp modes can be clearly seen in the wavelength range of $600 \mathrm{~nm}$ to $625 \mathrm{~nm}$. The integrated lasing intensity versus pump pulse energy is plotted in Figure $4 \mathrm{~b}$ demonstrating a nonlinear increase of the emission intensity. The lasing threshold in this case was estimated of $20 \mu \mathrm{J} / \mathrm{mm}^{2}$. The best full width at half maximum (FWHM) of the lasing modes was determined as $0.2 \mathrm{~nm}$. This led to a $\mathrm{Q}$-factor, defined as $\mathrm{Q}=\lambda / \Delta \lambda$, of approximately 3000 which is approximately three times higher of a solid state ring laser [17]. Note that, this value was limited by the spectrometer resolution.
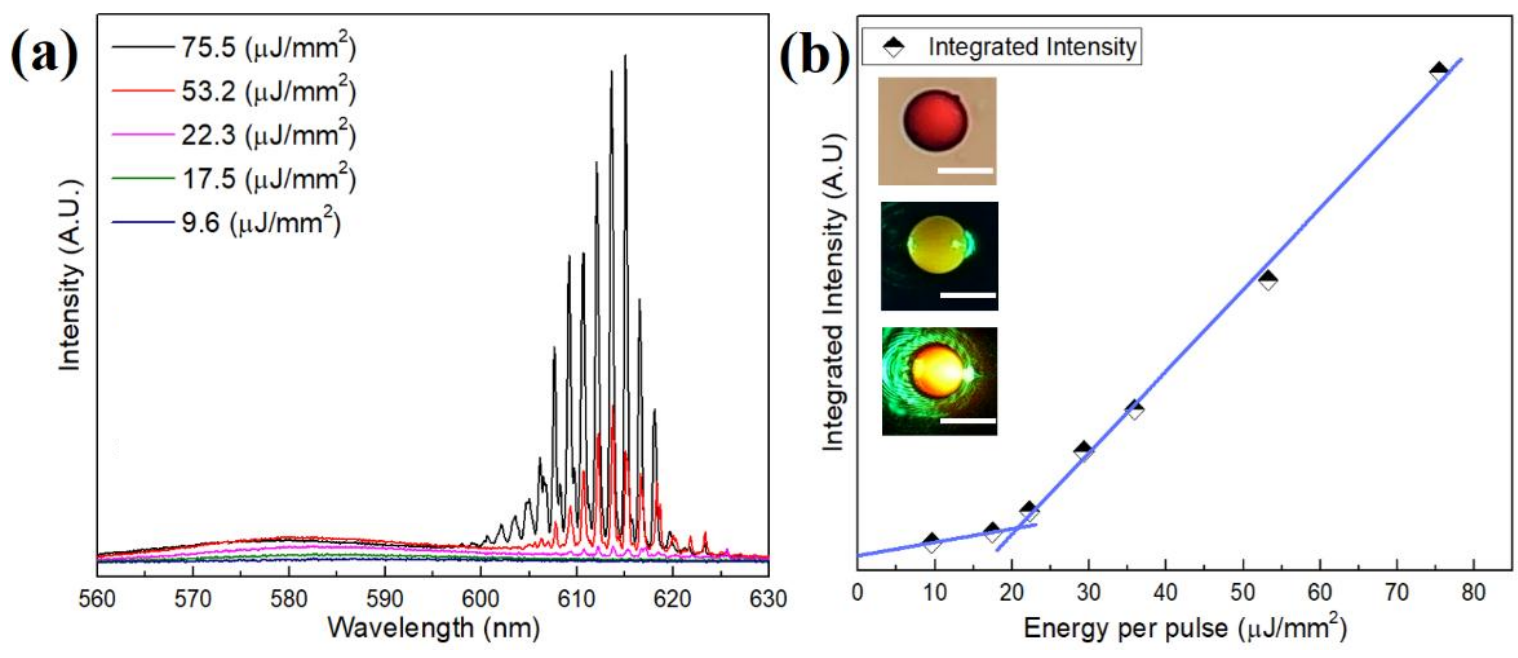

Figure 4. (a) Emission spectra of a $44 \mu \mathrm{m}$ dye-doped OVA microsphere,

(b) the nonlinear increase of the emission intensity with respect to pump pulse energy. The scale bar is $50 \mu \mathrm{m}$.

The lasing mechanism of the dye doped OVA microspheres is attributed to the confinement of light by the total internal reflection at the boundary between the microsphere and the surrounding environment. Technically speaking, a whispering gallery mode laser emission can be achieved when the path length of the light trapped inside the cavity equal integer times of its wavelength [18] $\left(2 \pi R n_{\text {eff }}=m \lambda_{m}\right)$. When the condition is satisfied, the lasing expected to occur at the equator of the spherical cavity.

In order to better verify the WGM mechanism, the free spectral ranges (FSR), the distance of two adjacent modes, of the microspheres with different sizes were investigated (Figure 5). As seen from the figure, the FSR decreases when the microlaser's dimeter increases. As shown in Figure 5 a-c the FSR of $22 \mu \mathrm{m}, 44 \mu \mathrm{m}$, and $67 \mu \mathrm{m}$ diameter microspheres are $3.5,1.6 \mathrm{~nm}$, and $1.2 \mathrm{~nm}$, respectively. According to Mie's scattering theory [19], at the resonant wavelength $\lambda$ of about $615 \mathrm{~nm}$, the calculated FSR = $\lambda / \pi \mathrm{n}_{1} \mathrm{D}$ (where $\mathrm{n}_{1}$ is the refractive index of ovalbumin of $\sim 1.55, \mathrm{D}$ is the diameter) for the three above microspheres are $3.5 \mathrm{~nm}, 1.6 \mathrm{~nm}$, and $1.3 \mathrm{~nm}$, respectively. The results show a good agreement between the theory and experimental observations. This again verifies that the lasing mechanism of the dye doped OVA microspheres are based on the WGM mechanism. Furthermore, as $\lambda$ is similar for different spheres, the FSR as a function of spherical cavity diameter should follow an $\alpha / \mathrm{D}$ function, where $\alpha$ is a constant. The FRS was well fitted with the function 78.65/D with $\alpha$ is equal to 78.65. 

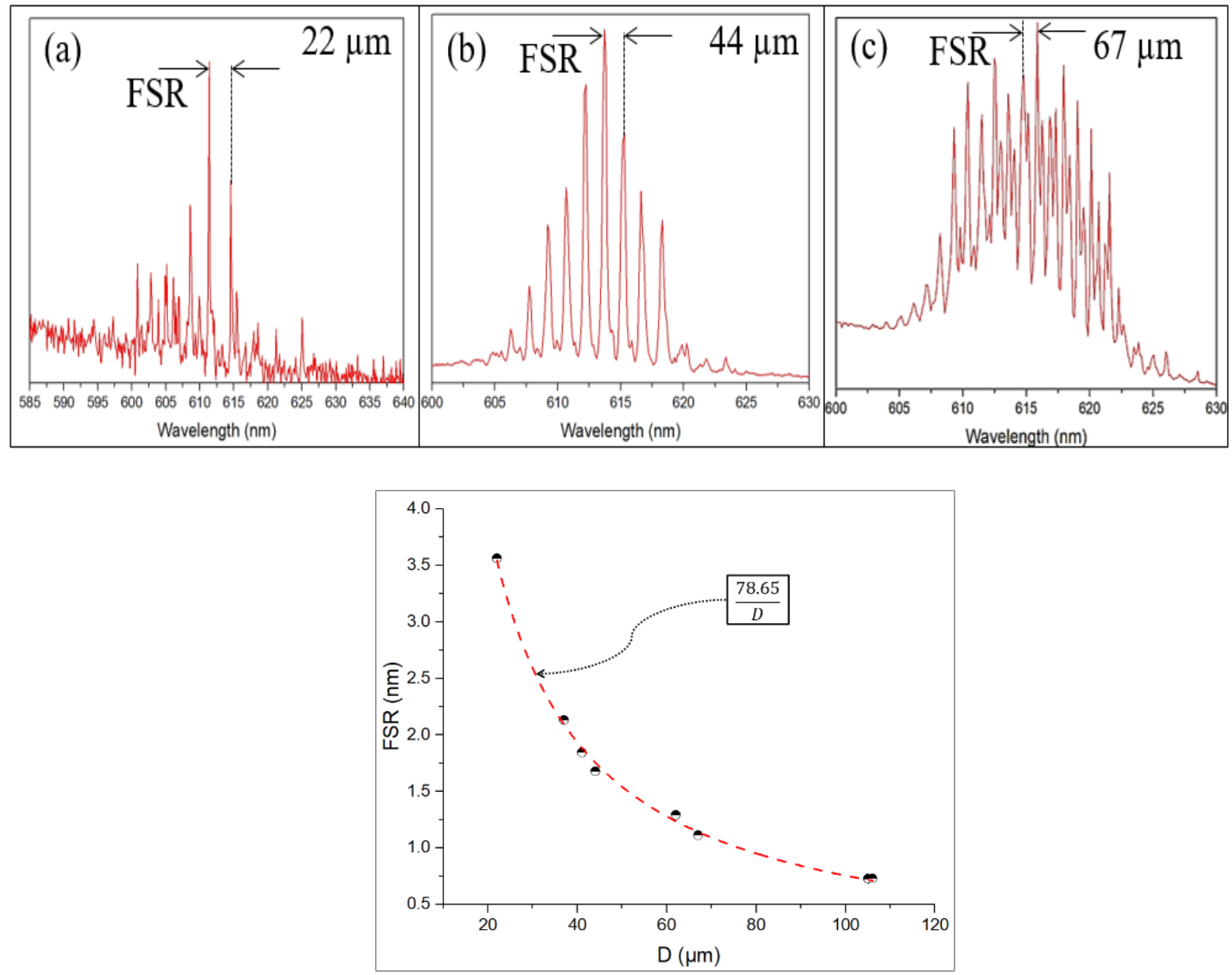

Figure 5. Spectra of three microspheres with different diameters of (a) $22 \mu \mathrm{m}$, (b) $44 \mu \mathrm{m}$, and (c) $67 \mu \mathrm{m}$. (d) Free spectral range as a function of diameter.

\section{Conclusion}

In this research, dye-doped OVA microspheres that generated WGM lasing with a simple heating process applying surface tension effect was successfully fabricated. The concentration of dye (RhB) was $0.5 \%$ in weight ratio to assure the biocompatibility of the microspheres. The micro-dye-doped OVA microspheres exhibited lasing emission at the threshold of $17.5 \mu \mathrm{J} / \mathrm{mm}^{2}$ and exhibited high Q-factor of 3000. FSR as a function of the spheres' diameter were also investigated showing a good agreement between experiment and theoretical calculation.

\section{Acknowledgement}

This work was supported by the International Center for Genetic Engineering and Biotechnology (ICGEB) through Grant No. CRP/VNM17-03. 


\section{References}

[1] V.D. Ta, Y. Wang, H. Sun, Microlasers Enabled by Soft- Matter Technology, Adv. Opt. Mater. 7 (2019) 1900057. https://doi.org/10.1002/adom.201900057.

[2] Y. Chen, X. Fan, Biological Lasers for Biomedical Applications, Adv. Opt. Mater. 7 (2019) 1900377. https://doi.org/10.1002/adom.201900377.

[3] V.D. Ta, S. Caixeiro, F.M. Fernandes, R. Sapienza, Microsphere Solid-State Biolasers, Adv. Opt. Mater. 5 (2017) 1-6. https://doi.org/10.1002/adom.201601022.

[4] M. Humar, A. Dobravec, X. Zhao, S.H. Yun, Biomaterial microlasers implantable in the cornea, skin, and blood, Optica. 4 (2017) 1080 - 1085. https://doi.org/10.1364/optica.4.001080.

[5] Y.L. Sun, Z.S. Hou, S.M. Sun, B.Y. Zheng, J.F. Ku, W.F. Dong, Q.D. Chen, H.B. Sun, Protein-Based Three-Dimensional Whispering-Gallery-Mode Micro-Lasers with Stimulus-Responsiveness, Sci. Rep. 5 (2015) 1-14. https://doi.org/10.1038/srep12852.

[6] J. Kovacs-Nolan, M. Phillips, Y. Mine, Advances in the Value of Eggs and Egg Components for Human Health, J. Agric. Food Chem. 53 (2005) 8421-8431. https://doi.org/10.1021/jf050964f.

[7] X. Wang, M. Bao, J. Wu, Y. Luo, L. Ma, Y. Wang, A. Zhang, C. He, H. Zhang, Characterization and Comparison of Ochratoxin A-Ovalbumin (OTA-OVA) Conjugation by Three Methods, J. Integr. Agric. 13 (2014) 1130-1136. https://doi.org/10.1016/S2095-3119(14)60767-X.

[8] S. Caixeiro, M. Gaio, B. Marelli, F.G. Omenetto, R. Sapienza, Silk-Based Biocompatible Random Lasing, Adv. Opt. Mater. 4 (2016) 998-1003. https://doi.org/10.1002/adom.201600185.

[9] C.S. Wang, T.Y. Chang, T.Y. Lin, Y.F. Chen, Biologically inspired flexible quasi-single-mode random laser: An integration of Pieris canidia butterfly wing and semiconductors, Sci. Rep. 4 (2014) 1-6. https://doi.org/10.1038/srep06736.

[10] D.S. Wiersma, Disordered photonics, Nat. Photonics. 7 (2013) 188-196. https://doi.org/10.1038/nphoton.2013.29.

[11] R.R. Da Silva, C.T. Dominguez, M. V. Dos Santos, R. Barbosa-Silva, M. Cavicchioli, L.M. Christovan, L.S.A. De Melo, A.S.L. Gomes, C.B. De Araújo, S.J.L. Ribeiro, Silk fibroin biopolymer films as efficient hosts for DFB laser operation, J. Mater. Chem. C. 1 (2013) 7181-7190. https://doi.org/10.1039/c3tc30903g.

[12] M.C. Gather, S.H. Yun, Single-cell biological lasers, Nat. Photonics. 5 (2011) 406-410. https://doi.org/10.1038/nphoton.2011.99.

[13] T. Reynolds, N. Riesen, A. Meldrum, X. Fan, J.M.M. Hall, T.M. Monro, A. François, Fluorescent and lasing whispering gallery mode microresonators for sensing applications, Laser Photonics Rev. 11 (2017) 1-20. https://doi.org/10.1002/lpor.201600265.

[14] V. Duong Ta, R. Chen, L. Ma, Y. Jun Ying, H. Dong Sun, Whispering gallery mode microlasers and refractive index sensing based on single polymer fiber, Laser Photonics Rev. 7 (2013) 133-139. https://doi.org/10.1002/lpor.201200074.

[15] M. Humar, S.H. Yun, Intracellular microlasers, Nat. Photonics. 9 (2015) 572-576. https://doi.org/10.1038/nphoton.2015.129.

[16] M. Schubert, A. Steude, P. Liehm, N.M. Kronenberg, M. Karl, E.C. Campbell, S.J. Powis, M.C. Gather, Lasing within Live Cells Containing Intracellular Optical Microresonators for Barcode-Type Cell Tagging and Tracking, Nano Lett. 15 (2015) 5647-5652. https://doi.org/10.1021/acs.nanolett.5b02491.

[17] M.C. Gather, S.H. Yun, Bio-optimized energy transfer in densely packed fluorescent protein enables near-maximal luminescence and solid-state lasers, Nat. Commun. 5 (2014) 1-8. https://doi.org/10.1038/ncomms6722.

[18] R. Chen, V.D Ta, H.D. Sun, Single mode lasing from hybrid hemispherical microresonators, Sci. Rep. 2 (2012) 244. https://doi.org/10.1038/srep00244.

[19] C.C. Lam, P.T. Leung, K. Young, Explicit asymptotic formulas for the positions, widths, and strengths of resonances in Mie scattering, J. Opt. Soc. Am. B. 9 (1992) 1585 - 1592.

https://doi.org/10.1364/JOSAB.9.001585. 